\title{
La eficiencia relativa del sector real versus la del sector financiero de la economía colombiana
}

\author{
The Relative Efficiency of the Real Sector \\ versus the Financial Sector of the \\ Colombian Economy
}

\section{A eficiência relativa do sector real vs. $O$ setor financeiro da economia colombiana}

Gloria Isabel Rodríguez Lozano * Michael Hernando Sarmiento Muñoz ${ }^{* *}$

DOI: https://doi.org/10.19053/01203053.v36.n64.2017.5258

Fecha de recepción: 8 de agosto de 2016

Fecha de aceptación: 25 de mayo de 2017

\footnotetext{
* Doctora en Ciencias Económicas de la Universidad Nacional de Colombia. Profesora asociada de la Facultad de Ciencias Económicas de la Universidad Nacional de Colombia. Correo electrónico: girodriguezl@unal.edu. co (D) http://orcid.org/0000-0001-6906-9223 Dirección Postal: Ciudad Universitaria Edificio 3A, Of. 308. Bogotá - Colombia

** Candidato a Magíster en Administración de la Universidad Nacional de Colombia. Especialista en Gerencia de Proyectos de la Universidad del Bosque e ingeniero industrial de la Universidad Nacional de Colombia. Bogotá - Colombia. Correo electrónico: mhsarmientom@unal.edu.co (iD) http://orcid.org/0000-0003-0198-6900
} 


\section{Resumen}

El objetivo de esta investigación es comparar las empresas del sector real de la economía y las entidades del sector financiero colombiano para el año 2014, en términos de eficiencia relativa, mediante la aplicación de la metodología Data Envelopment Analysis (DEA). Para un país como Colombia, perteneciente a las economías emergentes, es importante la realización de este estudio para determinar hasta dónde estos dos sectores son eficientes o ineficientes, dada la importancia que tienen en la generación de desarrollo y en la disminución de las desigualdades sociales. Se toman como fuente de información la Superintendencia de Sociedades y la Superintendencia Financiera. Se analizaron 26.799 empresas del sector real y 254 entidades del sector financiero, lo que dio como resultado mejores indicadores para el sector financiero y, dentro de este, se destacó la industria aseguradora. Sin embargo, es necesario que estos dos sectores mejoren su desempeño, ya que solo el 2,6 \% de las empresas y entidades estudiadas es eficiente.

Palabras clave: Data Envelopment Analysis (DEA), eficiencia relativa, sector real, sector financiero.

Clasificación JEL: M11, D24, G14, E01, C38, D57. 
Apuntes CENES Volumen 36, Número 64

julio - diciembre 2017. Págs. 111-138

\section{Abstract}

The objective of this research is to compare the companies of the real sector of the economy and the entities of the Colombian financial sector for the year 2014, in terms of relative efficiency, through the application of the Data Envelopment Analysis (DEA) methodology. For a country like Colombia, belonging to the emerging economies, it is important to conduct this study to determine how far these two sectors are efficient or inefficient, given the importance they have in generating development and reducing social inequalities. We take as source of information the Superintendencia de Sociedades and the Superintendencia Financiera. 26,799 companies in the real sector and financial sector entities 254 were analyzed resulting in better indicators for the financial sector and within this, the insurance industry stands. However it is necessary that these two sectors improve their performance to the extent that only $2.6 \%$ of studied companies and entities are efficient.

Keywords: Data Envelopment Analysis (DEA), relative efficiency, real sector, financial sector. 


\section{Resumo}

O objetivo desta pesquisa é comparar empresas do setor real da economia e as entidades do sector financeiro na Colômbia em 2014, em termos de eficiência relativa; através da aplicação da metodologia de Análise Envoltória de Dados (DEA). Para um país como a Colômbia, pertencente a economias emergentes, é importante para levar a cabo este estudo para determinar o quão longe esses dois setores são eficientes ou ineficientes; dada a importância na geração de desenvolvimento e redução das desigualdades sociais. É tomado como fonte de informação da Superintendencia de Sociedades e da Superintendencia Financeira. 26,799 empresas do setor real e entidades do sector financeiro 254 foram analisados. Resultando em melhoria de indicadores para o sector financeiro e dentro desta, a indústria de seguros está. No entanto, é necessário que estes dois sectores melhorar seu desempenho como apenas $2,6 \%$ das empresas e entidades estudadas são eficientes.

Palavras-chave: Data Envelopment Analysis (DEA), relativa eficiência, o setor real, Setor Financeiro. 
Apuntes CENES Volumen 36, Número 64

julio - diciembre 2017. Págs. 111-138

\section{INTRODUCCIÓN}

La noción de eficiencia tiene su origen en el término latino efficientia y hace referencia a la habilidad de contar con algo o alguien para obtener un resultado. En términos del concepto de eficiencia y su utilidad, y a manera de ejemplo, tenemos que para Chiavenato (2004, p. 52) eficiencia "significa utilización correcta de los recursos (medios de producción) disponibles. Puede definirse mediante la ecuación $E=P / R$, donde $P$ son los productos resultantes y R los recursos utilizados". Según Koontz y Weihrich (2004, p. 14), la eficiencia es "el logro de las metas con la menor cantidad de recursos". Mientras que para Robbins y Coulter (2005, p. 7), la eficiencia consiste en "obtener los mayores resultados con la mínima inversión". Ahora bien, para De Oliveira (2002, p. 20), la eficiencia significa "operar de modo que los recursos sean utilizados de forma más adecuada". Específicamente, para los economistas Samuelson y Nordhaus (2002, p.4), "eficiencia significa utilización de los recursos de la sociedad de la manera más eficaz posible para satisfacer las necesidades y los deseos de los individuos". Según Mankiw (2004, p.4), la eficiencia es la "propiedad según la cual la sociedad aprovecha de la mejor manera posible sus recursos escasos"; mientras que Andrade (2005, p. 253) la define como “expresión que se emplea para medir la capacidad o cualidad de actuación de un sistema o sujeto económico, para lograr el cumplimiento de objetivos determinados, minimizando el empleo de recursos". 
Para lograr la medición de la eficiencia se consideran dos grupos de técnicas: paramétricas y no paramétricas. El primer grupo se apoya fundamentalmente en métodos econométricos, que permiten hacer una estimación de valores desconocidos de los parámetros de la relación de producción y efectuar diversos contrastes respecto a la validez del modelo (Rodríguez, 2016). El segundo grupo hace la medición de la eficiencia comparando cada entidad estudiada con las entidades restantes, mediante una combinación lineal, para obtener una frontera eficiente compuesta por indicadores de cada una de las entidades, con técnicas no estadísticas de programación matemática.

En este segundo grupo aparece el análisis envolvente de datos (Data Envelopment Analysis DEA, por sus siglas en inglés), cuyo modelo inicial se denominó modelo CCR en honor a sus creadores Charnes, Cooper y Rhodes, (1978). Cabe anotar que DEA es una metodología de frontera utilizada para medir la eficiencia relativa.

Koopmans (1951) define la eficiencia como una relación de salida/entrada, para la que no es posible aumentar su salida o reducir su entrada sin una reducción simultánea de su opuesto dividendo o divisor. Desde su aporte conceptual a lo que significa la eficiencia técnica, se han venido generando contribuciones para lograr medir de mejor manera la eficiencia de las organizaciones. De tal manera que en el presente, DEA permite la medición de la eficiencia relativa de unidades organizacionales denominadas Decision Making Units (DMU por sus siglas en inglés), donde hay presencia de complejidad, pues existen múltiples entradas y múltiples salidas. Con esta técnica es posible la optimización de cada una de las unidades organizacionales, para obtener un grupo que forma la frontera de las que son eficientes y evi-denciar las que no lo son. Es necesario aclarar que la acepción de unidades organizacionales (DMU) se prefiere, dado que representa a todo tipo de or-ganizaciones donde se toman decisio-nes en su interior (Charnes, Cooper, Golany, Seiford \& Stutz, 1984).

En este contexto es relevante medir la eficiencia del sector real de la economía, puesto que son las entidades que lo componen las que permiten a los individuos y a otras empresas satisfacer sus necesidades con bienes o servicios que ofertan a sus consumidores. El bienestar de un país puede ser medido a través de la satisfacción que obtienen sus ciudadanos al poder obtener satisfactores en el mercado que garanticen un estilo de vida digno y libre. De ahí la importancia de que los recursos que se utilizan en la producción de bienes o los esfuerzos en la prestación de los servicios sean adecuados, es decir, eficientes. 
Pero también es necesario reconocer que estas entidades que componen el sector real de la economía requieren de capital que les permita financiarse para garantizar su operación, y son precisamente las entidades que componen el sector financiero las que tienen la facultad de proporcionarlo.

De tal manera que esta investigación presenta los resultados de la medición de la eficiencia relativa, indicadores DEA, tanto de las empresas del sector real de la economía colombiana, como de las entidades pertenecientes al sector financiero colombiano para el año 2014, haciendo una comparación de estos resultados.

\section{REVISIÓN BIBLIOGRÁFICA}

En el ámbito mundial, la metodología DEA ha sido utilizada para medir la eficiencia de los bancos, componen-te fundamental del sector financiero, como es el caso de los bancos vietnamitas, en donde un estudio reveló, para el período de 2000-2014, el impacto que la rentabilidad y los efectos de reformas hechas por el gobierno tuvieron en la eficiencia de los mismos (Nguyen, Nghiem, Roca \& Sharma, 2016); así como el caso de Ghana, en el que mediante el uso de DEA se determinó la relación entre eficiencia y competencia para los bancos de este país y cuyo fruto fue la recomendación de enfocar los esfuerzos en la mejora de la competitividad a través de la reducción de las tasas de interés y el acceso a los créditos para impactar positivamente en el crecimiento económico (Alhassan \& Ohene-Asare, 2016).

Para los países asiáticos del oriente de este continente se ha usado la técnica para examinar la relación entre la eficiencia de los bancos comerciales, la gobernabilidad del país y la regulación existente en los mercados financieros (Chan \& Abd, 2016). Para el caso latinoamericano, Seffino y Hoyos (2016) usaron DEA para medir la eficiencia de entidades bancarias argentinas e identificaron los elementos que permitieron que cada una de estas entidades alcanzara su respectivo desempeño, el resultado permitió vislumbrar una mejora en la productividad total.

En economías emergentes, la aplicación de DEA respecto al nivel de desarrollo económico de las provincias chinas mostró la necesidad de disminuir la disparidad que existe en estas regiones, a pesar de que sus oportunidades para mejorar son distintas; en las provincias ubicadas en la costa debería enfocarse en la innovación, mientras que en las provincias occidentales, en implementar estrategias de desarrollo (Li \& Zhao 2015). También el uso de la técnica permitió evaluar la eficiencia de la industria de alta tecnología en el mismo país para 2012, donde se evidenció el impacto que tienen los efectos de agentes ambientales no contro- 
lados para el desarrollo de su actividad económica (Jiang, Jiang, Wu, Liao \& Xu, 2015).

Škare y Rabar (2016) usan la técnica para la obtención de la eficiencia macroeconómica de los países, con el fin de entender las diferencias que existen en su desarrollo y crecimiento económico. Adler, Yazhemsky y Tarverdyan (2010) proporcionan en su investigación un marco que combina aspectos sociales, ambientales y económicos, para visualizar los países a través de la eficiencia relativa, respecto al uso que dan a sus recursos internos con el propósito de cumplir los objetivos de desarrollo del milenio (ODM).

Cronjé y Beer (2010) señalan que el desempeño financiero de los bancos, expresado en términos del rendimiento sobre activos (ROA), muestra que este se ajusta de acuerdo con la eficiencia de los ingresos y gastos, y tiene impacto directo en los beneficios futuros esperados. Para el caso latinoamericano, Chortareas, Garza-García y Girardone (2012) determinaron la eficiencia relativa de las observaciones de la banca mexicana, que corroboró los problemas más marcados en el continente: la falta de disponibilidad de créditos con tasas de interés bajas, a pesar de las reformas realizadas al sector financiero.

Para Europa, Thanassoulis, Boussofiane y Dyson (1995) evaluaron el desempeño de unidades organizativas como sucursales bancarias y escuelas en Inglaterra, utilizando DEA y el análisis de relaciones como herramienta alternativa, y concluyeron que la primera metodología arrojó resultados más apegados a la realidad. Para el caso de estudio, en Francia, Wanga, Lu y Liu (2014) investigaron la asociación entre el desempeño de las sociedades de cartera bancarias (BHC) y su capital intelectual (IC), a partir de la construcción de un modelo de DEA de dos etapas. La industria de las tarjetas de crédito también ha sido estudiada mediante DEA, por Saberi, Mirtalaie, Hussain, Azadeh, Hussain y Ashjari (2013), quienes, a través de datos para la Unión Europea, mejoraron las decisiones en línea. El sector bancario internacional, con sede en Austria, fue estudiado por Hahn (2009).

Ahora bien, en Canadá, Estrada, Song, Kim, Namnb y Kang (2009) estudiaron las sucursales de los bancos.

En Colombia se han hecho mediciones de eficiencia r elativa, tomando como salidas cuentas de los estados financieros reportados por empresas a la Superintendencia de Sociedades.

Fontalvo, Mendoza y Visbal (2015) determinaron la eficiencia para 32 empresas de Barranquilla afiliadas a la Alianza Empresarial para un Comercio Seguro, donde solo el 28 $\%$ de dichas organizaciones era eficiente. Moreno, López 
y Díaz (2014), por su parte, analizaron la eficiencia y productividad de cuatro actividades económicas de la construcción colombiana. Rodríguez (2003), para el caso de las empresas de alimentos y venta de vehículos, determinó cuáles organizaciones se mantuvieron más eficientes a través del tiempo; y Cayón y Sarmiento (2011) obtuvieron las eficiencias relativas del sector de calzado, vinculando el valor económico agregado (EVA), para determinar el factor de la fórmula que permite el cálculo de este indicador y poder buscar su maximización.

DEA también ha sido utilizado para medir la eficiencia relativa de diversas organizaciones (Vargas, 2016), dedicadas a distintas actividades económicas. Sin embargo, además ha sido utilizada para medir el desarrollo económico y crecimiento en Colombia, desde enfoques como la pobreza, la igualdad y la seguridad (Cotte, 2012), y para determinar el impacto que tienen la corrupción y la inseguridad, cuando factores clave como el desarrollo, los recursos naturales y la inestabilidad política afectan el crecimiento económico del país (Poveda, 2013). Adicionalmente, para establecer la eficiencia de costos en el sistema bancario en Colombia, donde DEA muestra los niveles de ineficiencia comparativamente con los estándares internacionales (Alma-zaRamírez, 2012).

\section{METODOLOGÍA}

La eficiencia relativa se introduce al tener en cuenta múltiples entradas y salidas (Farrell, 1957), con el fin de comparar diferentes unidades de decisión.

Fueron Charnes, Cooper y Rhodes (1978), quienes propusieron que cada una de las unidades organizacionales (DMU) adoptara un conjunto de pesos para cada entrada y salida, que fuera el más favorable respecto a las otras unidades. De tal manera que, si se propone maximizar la eficiencia de una unidad organizacional específica, es partiendo del hecho de que la eficiencia de todas las unidades estudiadas sea menor o igual que 1 .

Algunas de las variables para tener en cuenta son los pesos de las unidades organizacionales, pues al buscar la solución se generan los pesos más favorables para la unidad específica. Este razonamiento dio lugar al desarrollo de dos modelos que tienen como propósito disminuir las entradas manteniendo estables las salidas, o maximizar las salidas con un determinado nivel de recursos. Uno de estos modelos es el desarrollado por Banker, Charnes y Cooper (1984), denominado VRS (Variable Returns to Scale), y el otro es el ya mencionado CCR o CRS (Constant Returns to Scale). Para el primero, los retornos a escala son variables y para el segundo modelo estos rendimientos 
son constantes. Adicionalmente, para cada uno de ellos existen dos clases de orientaciones: a las entradas y a las salidas.

DEA determina la medida de desempeño de cada unidad organizacional máxima relativa a las otras unidades, obteniendo los pesos de cada una de ellas, y hecho esto, se establecen las unidades organizacionales que conforman la frontera eficiente (Charnes, Cooper, Lewin \& Seiford, 1994). Otra de las bondades de esta herramienta es la identificación de las unidades organizacionales ineficientes.

Para obtener la eficiencia relativa de las empresas del sector real de la economía y de las entidades pertenecientes al sector financiero colombiano, se establecieron como fuentes primarias de información los estados financieros de las organizaciones que reportan dicha información a las entidades que las supervisan: la Superintendencia de Sociedades y la Superintendencia Financiera de Colombia. La Superintendencia de Sociedades es un organismo técnico, adscrito al Ministerio de Comercio, Industria y Turismo, mediante el cual el presidente de la República ejerce la inspección, la vigilancia y el control de las sociedades mercantiles que componen el sector real de la economía colombiana. Esta Superintendencia mantiene el registro de la información financiera: balance general y estado de resultados. Cada empresa que debe reportar su información, debe también aportar su CIIU: Clasificación Industrial Internacional Uniforme de todas las actividades económicas. Esta clasificación consta de 21 secciones, cada una de estas secciones tiene divisiones, grupos y clases.

La Sección A: agricultura, ganadería, caza, silvicultura y pesca, tiene tres divisiones que están subdivididas en 13 grupos que, a su vez, agrupan a 36 clases.

La Sección B: explotación de minas y canteras, tiene cinco divisiones, 11 grupos y 17 clases.

La Sección C: industrias manufactureras, tiene 24 divisiones, 76 grupos y 151 clases.

La Sección D: suministro de electricidad, gas, vapor y aire acondicionado, tiene una división, tres grupos y seis clases.

La Sección E: distribución de agua, evacuación y tratamiento de aguas residuales, gestión de desechos y actividades de saneamiento ambiental, tiene cuatro divisiones, seis grupos y ocho clases.

La Sección F: construcción, tiene tres divisiones, ocho grupos y 12 clases.

La Sección G: comercio al por mayor y al por menor; reparación de vehícu- 
los automotores y motocicletas; tiene tres divisiones, 20 grupos y 58 clases.

La Sección H: transporte y almacenamiento; tiene cinco divisiones, 11 grupos y 22 clases.

La Sección I: alojamiento y servicios de comida; tiene dos divisiones, siete grupos y 15 clases.

La Sección J: información y comunicaciones; tiene seis divisiones, 13 grupos y 23 clases.

La Sección K: actividades financieras $\mathrm{y}$ de seguros, tiene tres divisiones, 11 grupos y 31 clases.

La Sección L: actividades inmobiliarias, tiene una división, dos grupos y dos clases.

La Sección M: actividades profesionales, científicas y técnicas; tiene siete divisiones, 14 grupos y 14 clases.

La Sección N: actividades de servicios administrativos y de apoyo, tiene seis divisiones, 19 grupos y 26 clases.

La Sección O: administración pública y defensa, planes de seguridad social de afiliación obligatoria, tiene una división, tres grupos y diez clases.

La Sección P: educación, tiene una división, cuatro grupos y 9 clases.

La Sección Q: actividades de atención de la salud humana y de asistencia social, tiene tres divisiones, nueve grupos y 12 clases.

La Sección R: actividades artísticas, de entretenimiento y recreación, tiene cuatro divisiones, cinco grupos y 17 clases.

La Sección S: otras actividades de servicios, tiene tres divisiones, seis grupos y 17 clases.

La Sección T: actividades de los hogares en calidad de empleadores; actividades no diferenciadas de los hogares individuales como productores de bienes y servicios para uso propio; tiene dos divisiones, tres grupos y tres clases.

La Sección U: actividades de organizaciones y entidades extraterritoriales, tiene una división, un grupo y una clase.

Por otro lado, la Superintendencia Financiera de Colombia surgió de la fusión de la Superintendencia Bancaria de Colombia con la Superintendencia de Valores, mediante el Decreto 4327 de 2005, modificado posteriormente por el Decreto 2555 de 2010, la Ley 1480 de 2011 y el Decreto 710 de 2012. La entidad es un organismo técnico adscrito al Ministerio de Hacienda y Crédito Público, con personería jurídica, autonomía administrativa y financiera y patrimonio propio, encar- 
gada de preservar la confianza pública y la estabilidad del sistema financiero; de mantener la integridad, la eficiencia y la transparencia del mercado de valores y demás activos financieros; y velar por el respeto a los derechos de los consumidores financieros y la debida prestación del servicio. En esta entidad se mantiene la trazabilidad de la información financiera (Balance General y Estado de Resultados) con el fin de dar firme cumplimiento a su misión.

La Superintendencia Financiera presenta la información de las entidades que vigila, agrupada de la siguiente manera:

- Establecimientos de crédito: banca de segundo piso, bancos, compañías de financiamiento comercial, cooperativas financieras, organismos cooperativos de grado superior.

- Industria aseguradora: compañías de seguros de vida, compañías de seguros generales, sociedades cooperativas de seguros y sociedades de capitalización.

- Intermediarios de valores y otros agentes: administradoras de sistemas de pago de bajo valor, almacenes generales de depósito y casa de cambio.

- Pensiones, cesantías y fiduciarias: fiduciarias, fondos de cesantías, fondos de pasivos pensionales, fondos de pensiones obligatorias, fondos de pensiones voluntarias, sociedades administradoras.

- Portafolios de inversión.

\section{MODELO DEA ESPECÍFICO}

Para esta investigación y dado que no fue posible establecer con certeza que los rendimientos a escala de las unidades estudiadas fuesen constantes, se optó por medir la eficiencia relativa mediante el modelo VRS, adicionalmente, y queriendo privilegiar la obtención de los mejores resultados se trabajó con la orientación a las salidas.

Para Rodríguez (2016), la formulación matemática parte de la consideración de un conjunto compuesto por $n$ DMUs, denotadas como DMU $(\mathrm{j}=1, \ldots, n)$; las cuales utilizan recur$\operatorname{sos} \mathrm{x}_{\mathrm{ij}}(\mathrm{i}=1, \ldots, m)$ y generan $\mathrm{s}$ outputs $\mathrm{y}_{\mathrm{rj}}(\mathrm{r}=1, \ldots \mathrm{s})$. Si los multiplicadores $\overline{\mathrm{v}}_{\mathrm{i}}$ $\overline{\mathrm{u}}_{\mathrm{r}}$ asociados con i entradas y $\mathrm{r}$ salidas respectivamente son conocidos con base en la teoría convencional beneficio/costo, la eficiencia $\bar{e}_{j}$ de $\mathrm{DMU}_{\mathrm{j}}$ se puede expresar como la proporción (ratio) de los pesos de las salidas y los pesos de las entradas, así:

$$
\sum_{\mathrm{r}} \overline{\mathrm{u}}_{r} \mathrm{y}_{r j} / \Sigma_{\mathrm{i}} \overline{\mathrm{v}}_{i} \mathrm{x}_{i j}
$$

Esta proporción salidas/entradas es la base para la medida estándar de la eficiencia. Charnes, Cooper y Rhodes (1978) proponen derivar apropiadamente los multiplicadores para que 
cada DMU obtenga la solución particular al problema de programación no lineal. Específicamente, si la DMUo bajo estudio, este modelo da solución al problema de programación fraccional para la medida de eficiencia técnica de esa DMUo:

$$
e_{\mathrm{o}}=\max \quad \sum_{r} \mathrm{u}_{r} \mathrm{y}_{r o} / \Sigma_{i} \mathrm{~V}_{i} \mathrm{x}_{i o}
$$

Sujeto a:

$$
\begin{gathered}
\sum_{\mathrm{r}} \mathrm{u}_{r} \mathrm{y}_{r j}-\Sigma_{\mathrm{i}} \mathrm{v}_{i} \mathrm{X}_{i j} \leq 0 \text {, para todo } \mathrm{j} \\
\mathrm{U}_{\mathrm{r}}, \mathrm{v}_{\mathrm{i}} \geq \mathcal{E}, \text { para todo } r, i
\end{gathered}
$$

Donde $\mathcal{E}$ es un valor no arquimediano designado estrictamente positivo.

El problema inicial fue transformado así:

$$
e_{\mathrm{o}}=\max \quad \Sigma_{\mathrm{r}} \mu_{\mathrm{r}} \mathrm{y}_{\mathrm{ro}}
$$

Sujeto a:

$$
\begin{gathered}
\sum_{i} \mathrm{~V}_{i} \mathrm{x}_{i o=1} \\
\sum_{\mathrm{r}} \mu_{\mathrm{r}} \mathrm{y}_{r j-} \sum_{i} \mathrm{~V}_{i} \mathrm{X}_{i j \leq 0,} \quad \forall j \\
\mu_{r}, v_{i} \geq \mathcal{E} \text { para todo } r, i
\end{gathered}
$$

Para el modelo VRS la formulación matemática es la siguiente:

$$
e_{\mathrm{o}}^{*}=\max \left[\sum_{r} u_{r} y_{r o}-u_{o}\right] / \Sigma_{i} v_{i} x_{i o}
$$

Sujeto a:

$$
\begin{aligned}
& \sum_{r} u_{r} y_{r j}-u_{o}-\Sigma_{i} v_{r} x_{i j} \leq 0 j=1, \ldots, n \\
& u_{r} \geq \mathcal{E}, \quad v_{i} \geq \mathcal{E},
\end{aligned}
$$

$u_{o}$ no restringida en signo

Con su equivalente en programación lineal:

$$
e_{\mathrm{o}}^{*}=\max \quad \Sigma_{r} \mu_{r} y_{r o}-\mu_{o}
$$

Sujeto a:

$\sum_{i} v_{i} x_{i o}=1$

$\Sigma_{r} \mu_{r} y_{r j}-\mu_{o}-\Sigma_{i} v_{i} x_{i o} \leq 0, \quad j=1, \ldots n$

$\mu_{r} \geq \mathcal{E}, r_{i} \geq \mathcal{E}$

$\mu_{o \text { irrestricta }}$

Los modelos DEA trabajan con $n$ unidades que toman decisiones (DMUs) que se quieren evaluar y cada una de ellas consumen variadas cantidades de $m$ diferentes recursos (imputs) para producir $s$ diferentes salidas (outputs). Así: $\mathrm{DMU}_{\mathrm{j}}$ asume $\mathrm{X}_{\mathrm{j}}=\left\{x_{i j}\right\}$ cantidades de entradas $(i=1, \ldots, m)$ y produce $\mathrm{Y}_{\mathrm{j}}=$ $\left\{y_{r j}\right\}$ cantidades de salidas $(r=1, \ldots, s)$. Adicionalmente se asume que $x_{i j}>0 \mathrm{y}$ $y_{i j}>0$. La matriz $s \times n$ de salidas se denota como Y. La matriz $m \times n$ de entradas es denotada por X. (Rodríguez, 2016).

VRS orientado a las entradas:

Primal $\quad \min$

$$
\theta, \lambda, s^{+}, s_{-} \quad Z_{0}=\theta-\mathcal{E} \cdot s^{+}-\varepsilon \cdot s^{-}
$$

Sujeto a:

$$
\begin{aligned}
& \mathrm{Y} \lambda-\mathrm{s}^{+}=\mathrm{Y}_{\mathrm{o}} \\
& \theta \mathrm{X}_{\mathrm{o}}-\mathrm{X} \lambda-\mathrm{s}^{-}=0
\end{aligned}
$$


$\lambda \geq 1$

$\lambda, \mathrm{s}^{+}, \mathrm{s}^{-} \geq 0$

Dual $\max \omega_{0}=\mu^{\mathrm{T}} Y_{\mathrm{o}}+\mathrm{u}_{\mathrm{o}}$

[7]

$\mu, \mathrm{v}$

Sujeto a:

$\mathrm{V}^{\mathrm{T}} \mathrm{X}_{\mathrm{o}}=1$

$\mu^{\mathrm{T}} \mathrm{Y}-\mathrm{v}^{\mathrm{T}} \mathrm{X}+\mathrm{u}_{\mathrm{o}} \leq 0$

$-\mu^{\mathrm{T}} \leq-\varepsilon \cdot$

$-\mathrm{v}^{\mathrm{T}} \leq-\varepsilon \cdot$

$\mathrm{u}_{\mathrm{o}}$ libre de signo

$\boldsymbol{\theta}$ : variable proporcional escalar de reducción aplicada a todas las entradas de la $\mathrm{DMU}_{\mathrm{o}}$ para mejorar eficiencia.

$\varepsilon$ : constante infinitesimal noarquimedia, permite la minimización sobre $\theta$.

$s^{+}, s^{-}$: variable de holgura.

$\overrightarrow{1}$ : vector de unos

Una DMU es eficiente si y solo si satisface las siguientes condiciones:

$\left.\theta^{*}=1 ; \mathrm{b}\right)$ todas las holguras son cero.

Una DMU es eficiente si y solo si $\omega_{\mathrm{o}}$ * $=\mathrm{Z}_{\mathrm{o}}^{*}=1$

VRS orientado a las salidas:

Primal $\quad \min$

$$
\varphi, \lambda, \mathrm{s}^{+}, \mathrm{s}^{-} \quad \mathrm{Z}_{\mathrm{o}}=\varphi-\varepsilon \cdot \mathrm{s}^{+}-\varepsilon \cdot \mathrm{s}^{-}
$$

Sujeto a:

$$
\begin{aligned}
\varphi \mathrm{Y}_{\mathrm{o}}-\mathrm{Y} \lambda+\mathrm{s}^{+} & =0 \\
\mathrm{X} \lambda-\mathrm{s}^{-} & =\mathrm{X}_{\mathrm{o}} \\
\lambda & =1 \\
\lambda, \mathrm{s}^{+}, \mathrm{s}^{-} & \geq 0
\end{aligned}
$$

Dual $\min \quad \mathrm{q}_{\mathrm{o}}=\mathrm{v}^{\mathrm{T}} \mathrm{X}_{\mathrm{o}}+\mathrm{v}_{\mathrm{o}} \quad[9]$

$$
\mu, \mathrm{v}, \mathrm{v}_{\mathrm{o}}
$$

Sujeto a:

$$
\begin{gathered}
\mu^{\mathrm{T}} Y_{\mathrm{o}}=1 \\
-\mu^{\mathrm{T} Y}+\mathrm{v}^{\mathrm{T} X}+\mathrm{v}_{\mathrm{o}} \geq 0 \\
\mu^{\mathrm{T}} \geq \varepsilon . \\
\mathrm{v}^{\mathrm{T}} \geq \varepsilon \cdot \\
\mathrm{v}_{\mathrm{o}} \text { libre en signo }
\end{gathered}
$$

Partiendo del hecho de que DEA es un modelo de frontera no paramétrico, en el cual no es necesario preestablecer la función de producción, y que las variables determinantes del modelo son los recursos (entradas) y lo que se obtiene del proceso de transformación de ellos (salidas), las variables utilizadas en esta investigación se muestran en la Tabla 1. 
Apuntes CENES Volumen 36, Número 64

julio - diciembre 2017. Págs. 111-138

Tabla 1. Variables DEA

\begin{tabular}{|l|l|}
\hline \multicolumn{1}{|c|}{ ENTRADAS } & \multicolumn{1}{c|}{ SALIDAS } \\
\hline Activo corriente & Ingresos operacionales \\
\hline Propiedades planta y equipo & Utilidad neta \\
\hline Pasivo no corriente & \\
\hline Patrimonio & \\
\hline
\end{tabular}

Para el año 2014 se encontraron 26.799 empresas pertenecientes al sector real de la economía colombiana, que reportaron su información financiera a la Superintendencia de Sociedades; todas ellas hicieron parte de esta investigación.

Para este mismo año se estableció que en la Superintendencia Financiera reportaron sus estados finan- cieros 254 entidades financieras, las cuales también hicieron parte de esta investigación.

\section{RESULTADOS}

\section{Para las empresas del sector real}

Para el sector real, 660 empresas fueron eficientes $(2,5 \%)$. La Tabla 2 y la Figura 1 muestran cómo están distribuidas.

Tabla 2. Empresas Eficientes Sectot Real

\begin{tabular}{|c|c|l|}
\hline $\begin{array}{c}\text { Número empresas } \\
\text { eficientes }\end{array}$ & Porcentaje & \multicolumn{1}{|c|}{ Sección } \\
\hline 268 & 40,6 & $\begin{array}{l}\text { G: comercio al por mayor y al por menor; reparación } \\
\text { de vehículos automotores y motocicletas }\end{array}$ \\
\hline 76 & 11,5 & C: industrias manufactureras \\
\hline 46 & 7,0 & F: construcción \\
\hline 44 & 6,7 & M: actividades profesionales, científicas y técnicas \\
\hline 39 & 5,9 & B: explotación de minas y canteras \\
\hline 38 & 5,8 & J: información y comunicaciones \\
\hline 32 & 4,8 & $\begin{array}{l}\text { N: actividades de servicios administrativos y de } \\
\text { apoyo }\end{array}$ \\
\hline 27 & 4,1 & A: agricultura, ganadería, caza, silvicultura y pesca \\
\hline
\end{tabular}


La eficiencia relativa del sector real versus la del sector financiero de la economía colombia

Gloria Isabel Rodríguez Lozano Michael Hernando Sarmiento Muñoz

\begin{tabular}{|l|l|l|}
\hline 26 & 3,9 & K: actividades financieras y de seguros \\
\hline 23 & 3,5 & L: actividades inmobiliarias \\
\hline 19 & 2,9 & H: transporte y almacenamiento \\
\hline 7 & 1,1 & $\begin{array}{l}\text { R: actividades artísticas, de entretenimiento y } \\
\text { recreación }\end{array}$ \\
\hline 6 & 0,9 & I: alojamiento y servicios de comida \\
\hline 4 & 0,6 & S: otras actividades de servicios \\
\hline 2 & 0,3 & $\begin{array}{l}\text { D: suministro de electricidad, gas, vapor y aire } \\
\text { acondicionado }\end{array}$ \\
\hline 2 & 0,3 & $\begin{array}{l}\text { Q: actividades de atención de la salud humana y de } \\
\text { asistencia social }\end{array}$ \\
\hline 1 & 0,2 & P: educación \\
\hline
\end{tabular}

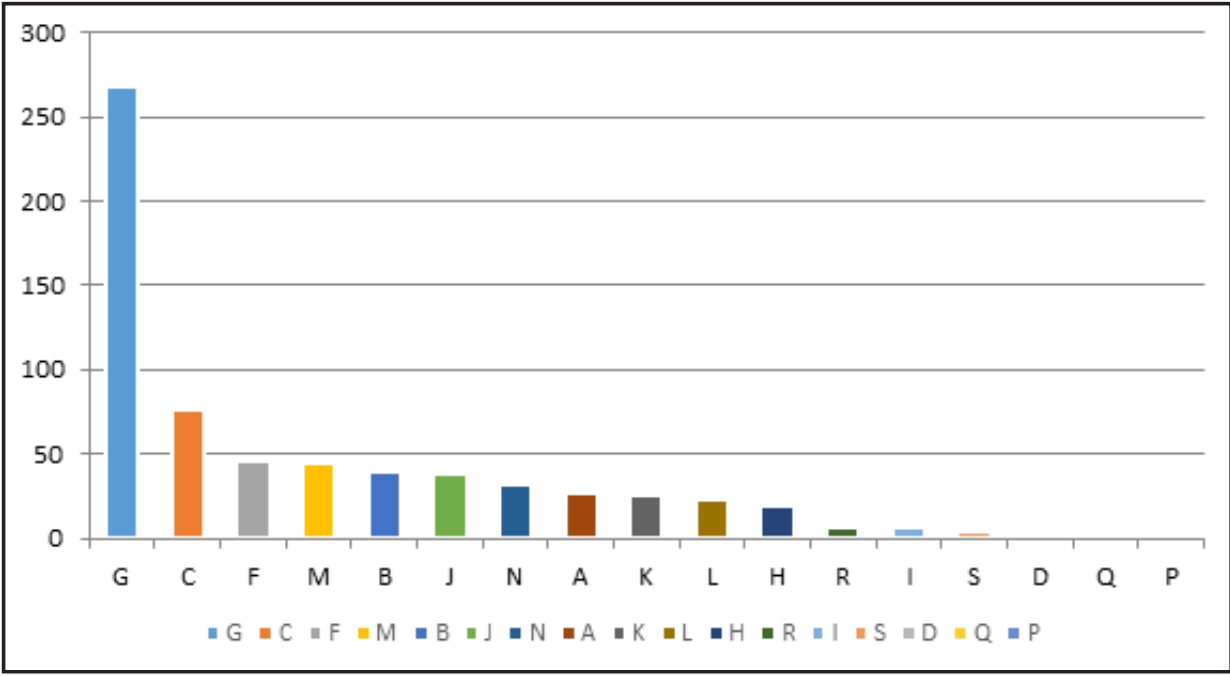

Figura 1. Empresas eficientes sector real

El $52.1 \%$ de las empresas eficientes del sector real pertenece al subsector del comercio al por mayor $\mathrm{y}$ al por menor $\mathrm{y}$ a las industrias manufactureras.
En la Tabla 3 se muestran las secciones que no tuvieron ninguna empresa eficiente. 
Apuntes CENES Volumen 36, Número 64

julio - diciembre 2017. Págs. 111-138

Tabla 3. Secciones sin Empresas Eficientes

\begin{tabular}{|c|l|}
\hline Sección & \multicolumn{1}{|c|}{ Descripción } \\
\hline E & $\begin{array}{l}\text { distribución de agua; evacuación y tratamiento de aguas residuales, gestión } \\
\text { de desechos y actividades de saneamiento ambiental }\end{array}$ \\
\hline 0 & $\begin{array}{l}\text { administración pública y defensa; planes de seguridad social de afiliación } \\
\text { obligatoria }\end{array}$ \\
\hline T & $\begin{array}{l}\text { actividades de los hogares individuales en calidad de empleadores; activida- } \\
\text { des no diferenciadas de los hogares individuales como productores de bienes } \\
\text { y servicios para uso propio }\end{array}$ \\
\hline U & actividades de organizaciones y entidades extraterritoriales \\
\hline
\end{tabular}

En la Tabla 4 se muestra la distribución de empresas eficientes de la sección G.

Tabla 4. Empresas Eficentes Sección G

\begin{tabular}{|c|c|l|}
\hline Clase & Número & \multicolumn{1}{c|}{ Descripción } \\
\hline G4645 & 20 & $\begin{array}{l}\text { Comercio al por mayor de productos farmacéuticos, medicinales, } \\
\text { cosméticos y de tocador. }\end{array}$ \\
\hline G4690 & 19 & Comercio al por mayor no especializado. \\
\hline G4664 & 18 & $\begin{array}{l}\text { Comercio al por mayor de productos químicos básicos, cauchos y } \\
\text { plásticos en formas primarias y productos químicos de uso agro- } \\
\text { pecuario. }\end{array}$ \\
\hline G4659 & 17 & Comercio al por mayor de otros tipos de maquinaria y equipo. \\
\hline G4663 & 15 & $\begin{array}{l}\text { Comercio al por mayor de materiales de construcción, artículos de } \\
\text { ferretería, pinturas, productos de vidrio, equipo y materiales de fonta- } \\
\text { nería y calefacción. }\end{array}$ \\
\hline G4669 & 13 & Comercio al por mayor de otros productos. \\
\hline G4511 & 12 & Comercio de vehículos automotores nuevos. \\
\hline G4711 & 10 & $\begin{array}{l}\text { Comercio al por menor en establecimientos no especializados con } \\
\text { surtido compuesto principalmente por alimentos, bebidas o tabaco. }\end{array}$ \\
\hline
\end{tabular}


La eficiencia relativa del sector real versus la del sector financiero de la economía colombia

Gloria Isabel Rodríguez Lozano Michael Hernando Sarmiento Muñoz

\begin{tabular}{|c|c|c|}
\hline G4530 & 9 & $\begin{array}{l}\text { Comercio de partes, piezas (autopartes) y accesorios (lujos) para } \\
\text { vehículos automotores. }\end{array}$ \\
\hline G4661 & 9 & $\begin{array}{l}\text { Comercio al por mayor de combustibles sólidos, líquidos, gaseosos } \\
\text { y productos conexos. }\end{array}$ \\
\hline G4620 & 8 & $\begin{array}{l}\text { Comercio al por mayor de materias primas agropecuarias; animales } \\
\text { vivos. }\end{array}$ \\
\hline G4731 & 8 & Comercio al por menor de combustible para automotores. \\
\hline G4771 & 8 & $\begin{array}{l}\text { Comercio al por menor de prendas de vestir y sus accesorios (incluye } \\
\text { artículos de piel) en establecimientos especializados. }\end{array}$ \\
\hline G4773 & 8 & $\begin{array}{l}\text { Comercio al por menor de productos farmacéuticos y medici- } \\
\text { nales, cosméticos y artículos de tocador en establecimientos } \\
\text { especializados. }\end{array}$ \\
\hline G4644 & 7 & Comercio al por mayor de aparatos y equipo de uso doméstico. \\
\hline G4651 & 7 & $\begin{array}{l}\text { Comercio al por mayor de computadores, equipo periférico y progra- } \\
\text { mas de informática. }\end{array}$ \\
\hline G4761 & 7 & $\begin{array}{l}\text { Comercio al por mayor de combustibles sólidos, líquidos, gaseosos } \\
\text { y productos conexos. }\end{array}$ \\
\hline G4541 & 6 & Comercio de motocicletas y de sus partes, piezas y accesorios. \\
\hline G4754 & 6 & $\begin{array}{l}\text { Comercio al por menor de electrodomésticos y gasodomésticos de } \\
\text { uso doméstico, muebles y equipos de iluminación. }\end{array}$ \\
\hline G4631 & 5 & Comercio al por mayor de productos alimenticios. \\
\hline G4649 & 5 & Comercio al por mayor de otros utensilios domésticos. \\
\hline G4652 & 4 & $\begin{array}{l}\text { Comercio al por mayor de equipo, partes y piezas electrónicos y de } \\
\text { telecomunicaciones. }\end{array}$ \\
\hline G4610 & 3 & Comercio al por mayor a cambio de una retribución o por contrata. \\
\hline G4641 & 3 & $\begin{array}{l}\text { Comercio al por mayor de productos textiles, productos confecciona- } \\
\text { dos para uso doméstico. }\end{array}$ \\
\hline G4642 & 3 & Comercio al por mayor de prendas de vestir. \\
\hline G4662 & 3 & Comercio al por mayor de metales y productos metaliferos. \\
\hline G4665 & 3 & Comercio al por mayor de desperdicios, desechos y chatarra. \\
\hline
\end{tabular}


Apuntes CENES Volumen 36, Número 64

julio - diciembre 2017. Págs. 111-138

\begin{tabular}{|c|c|c|}
\hline G4772 & 3 & $\begin{array}{l}\text { Comercio al por menor de todo tipo de calzado y artículos de cuero y } \\
\text { sucedáneos del cuero en establecimientos especializados. }\end{array}$ \\
\hline G4799 & 3 & $\begin{array}{l}\text { Otros tipos de comercio al por menor no realizado en establecimien- } \\
\text { tos, puestos de venta o mercados. }\end{array}$ \\
\hline G4632 & 2 & Comercio al por mayor de bebidas y tabaco. \\
\hline G4643 & 2 & Comercio al por mayor de calzado. \\
\hline G4719 & 2 & $\begin{array}{l}\text { Comercio al por menor en establecimientos no especializados, con } \\
\text { surtido compuesto principalmente por productos diferentes de ali- } \\
\text { mentos (víveres en general), bebidas y tabaco. }\end{array}$ \\
\hline G4729 & 2 & $\begin{array}{l}\text { Comercio al por menor de otros productos alimenticios, en estableci- } \\
\text { mientos especializados. }\end{array}$ \\
\hline G4752 & 2 & $\begin{array}{l}\text { Comercio al por menor de artículos de ferretería, pinturas y productos } \\
\text { de vidrio en establecimientos especializados. }\end{array}$ \\
\hline G4759 & 2 & $\begin{array}{l}\text { Comercio al por menor de otros artículos domésticos en estableci- } \\
\text { mientos especializados. }\end{array}$ \\
\hline G4791 & 2 & Comercio al por menor realizado a través de internet. \\
\hline G4520 & 2 & Mantenimiento y reparación de vehículos automotores. \\
\hline G4542 & 2 & Mantenimiento y reparación de motocicletas y de sus partes y piezas. \\
\hline G4653 & 1 & Comercio al por mayor de maquinaria y equipo agropecuarios. \\
\hline G4722 & 1 & $\begin{array}{l}\text { Comercio al por menor de leche, productos lácteos y huevos, en es- } \\
\text { tablecimientos especializados. }\end{array}$ \\
\hline G4732 & 1 & $\begin{array}{l}\text { Comercio al por menor de lubricantes (aceites, grasas), aditivos y } \\
\text { productos de limpieza para vehículos automotores. }\end{array}$ \\
\hline G4741 & 1 & $\begin{array}{l}\text { Comercio al por menor de computadores, equipos periféricos, pro- } \\
\text { gramas de informática y equipos de telecomunicaciones en estable- } \\
\text { cimientos especializados. }\end{array}$ \\
\hline G4751 & 1 & $\begin{array}{l}\text { Comercio al por menor de productos textiles en establecimientos es- } \\
\text { pecializados. }\end{array}$ \\
\hline G4762 & 1 & $\begin{array}{l}\text { Comercio al por menor de artículos deportivos, en establecimientos } \\
\text { especializados. }\end{array}$ \\
\hline G4774 & 1 & $\begin{array}{l}\text { Comercio al por menor de otros productos nuevos en establecimien- } \\
\text { tos especializados. }\end{array}$ \\
\hline G4792 & 1 & $\begin{array}{l}\text { Comercio al por menor realizado a través de casas de venta o por } \\
\text { correo. }\end{array}$ \\
\hline
\end{tabular}


Para la Sección C, dentro de las empresas eficientes no hay ninguna que pertenezca a las siguientes divisiones:

23: Fabricación de otros productos minerales no metálicos.

26: Fabricación de productos informáticos, electrónicos y ópticos.
27: Fabricación de aparatos y equipo eléctrico.

30: Fabricación de otros tipos de equipo de transporte.

Adicionalmente, para esta misma Sección, en los grupos que muestra la Tabla 5 tampoco hay empresas eficientes.

Tabla 5. Grupos sin Empresas Eficientes de la Sección C

\begin{tabular}{|c|l|}
\hline GRUPO & \multicolumn{1}{|c|}{ DESCRIPCIÓN } \\
\hline 103 & Elaboración de aceites y grasas de origen vegetal y animal. \\
\hline 105 & $\begin{array}{l}\text { Elaboración de productos de molinería, almidones y productos derivados del al- } \\
\text { midón. }\end{array}$ \\
\hline 107 & Elaboración de azúcar y panela. \\
\hline 109 & Elaboración de alimentos preparados para animales. \\
\hline 142 & Fabricación de artículos de piel. \\
\hline 143 & Fabricación de artículos de punto y ganchillo. \\
\hline 151 & $\begin{array}{l}\text { Curtido y recurtido de cueros; fabricación de artículos de viaje, bolsos de mano } \\
\text { y artículos similares, y fabricación de artículos de talabartería y guarnicionería, } \\
\text { adobo y teñido de pieles. }\end{array}$ \\
\hline 164 & Fabricación de recipientes de madera. \\
\hline 169 & $\begin{array}{l}\text { Fabricación de otros productos de madera; fabricación de artículos de corcho, } \\
\text { cestería y espartería. }\end{array}$ \\
\hline 182 & Producción de copias a partir de grabaciones originales. \\
\hline 191 & Fabricación de productos de hornos de coque. \\
\hline 203 & Fabricación de fibras sintéticas y artificiales. \\
\hline 252 & Fabricación de armas y municiones. \\
\hline 292 & $\begin{array}{l}\text { Fabricación de carrocerías para vehículos automotores; fabricación de remolques } \\
\text { y semirremolques. }\end{array}$ \\
\hline
\end{tabular}




\begin{tabular}{|c|l|}
\hline 312 & Fabricación de colchones y somieres. \\
\hline 321 & Fabricación de joyas, bisutería y artículos conexos. \\
\hline 322 & Fabricación de instrumentos musicales. \\
\hline 323 & Fabricación de artículos y equipo para la práctica del deporte. \\
\hline 324 & Fabricación de juegos, juguetes y rompecabezas. \\
\hline 325 & $\begin{array}{l}\text { Fabricación de instrumentos, aparatos y materiales médicos y odontológicos (in- } \\
\text { cluido mobiliario). }\end{array}$ \\
\hline 332 & Instalación especializada de maquinaria y equipo industrial. \\
\hline
\end{tabular}

\section{Para las entidades pertenecientes al sector financiero}

Se estableció que 42 entidades fueron eficientes (17\%). La Tabla 6 y la Figura 2 muestran cómo están distribuidas.

Tabla 6. Entidades Eficiente Sector Financiero

\begin{tabular}{|c|c|l|}
\hline $\begin{array}{c}\text { Número entida- } \\
\text { des eficientes }\end{array}$ & Porcentaje & \multicolumn{1}{c|}{ Grupo } \\
\hline 10 & 24 & Seguros de vida \\
\hline 8 & 19 & Corredores de seguros \\
\hline 6 & 14 & Seguros generales \\
\hline 5 & 12 & Bancos \\
\hline 3 & 7 & Fiduciaria \\
\hline 2 & 5 & Administradora fondos de pensiones \\
\hline 2 & 5 & Banca de segundo piso \\
\hline 2 & 5 & Fondos pensiones obligatorias \\
\hline 1 & 2 & Almacenes generales de depósito \\
\hline 1 & 2 & Corporación financiera \\
\hline 1 & 2 & Pasivos pensionales \\
\hline 1 & 2 & Sociedades de capitalización \\
\hline
\end{tabular}




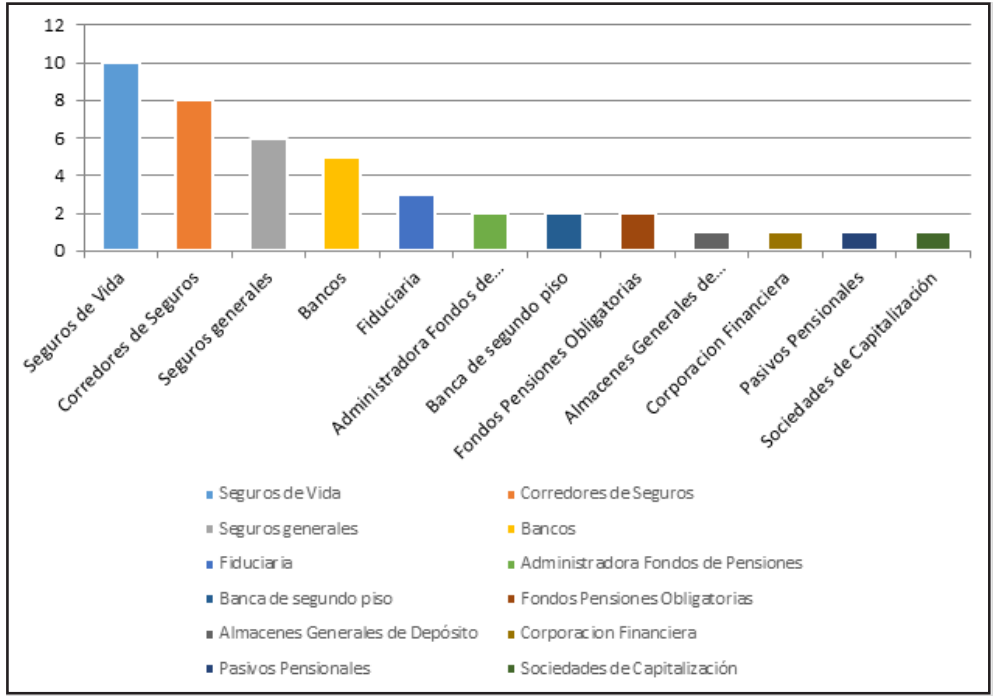

Figura 2. Entidades eficientes sector financiero

De tal manera que la industria aseguradora tiene una participación del $60 \%$ en las entidades eficientes, haciendo de este subsector económico el contributario más importante en cuanto a número de entidades eficientes.

Dentro de los establecimientos de crédito no hay compañías de financiamiento comercial ni cooperativas financieras eficientes. Para la industria aseguradora no existen eficientes en la categoría de sociedades cooperativas de seguros. En intermediarios de valores y otros agentes no hay administradoras de sistemas de pagos de bajo valor eficientes ni casas de cambio. Tampoco hay entidades eficientes en fondos de cesantías, fondos de pensiones voluntarias, sociedades administradoras ni en fondos de inversión.

\section{Comparación}

Del análisis de los pesos se establece que para el sector real, la eficiencia radica prioritariamente en el mejor aprovechamiento de los recursos provenientes del activo corriente en un $80 \%$ $\mathrm{y}$ de propiedades planta y equipo en un $18 \%$; respecto a las salidas se determinó que este sector estriba su eficiencia preferentemente en los ingresos operacionales con $94 \%$. Para el sector financiero, los resultados muestran que la eficiencia radica en el mejor aprovechamiento de los recursos provenientes del activo corriente en un $60 \%$ y de propiedades planta y equipo en un $21 \%$; respecto a las salidas, la eficiencia está en los ingresos operacionales en un nivel del $66 \%$. 
De tal manera que se establece que el sector financiero tiene una estructura más adecuada, tanto en el aprovechamiento de los recursos como en la estructura de eficiencia de las salidas, pues no es aconsejable tener un nivel tan alto (del $80 \%$ ) en una sola de las cuatro fuentes de entradas $y$, de igual manera, tener una sola de las dos salidas con una representación bien cercana al $100 \%$.

\section{CONCLUSIONES}

El sector financiero tiene un mejor comportamiento respecto al porcentaje de empresas eficientes frente al sector real (17\% frente a 2,5\%). En el sector real, dos de 21 secciones tienen más de la mitad de las empresas eficientes. Secciones tan importantes como la construcción y la explotación de minas y canteras, apenas tienen una representatividad menor al $13 \%$. Hay cuatro secciones cuyo aporte es del $0 \%$, son las secciones $\mathrm{E}, \mathrm{O}, \mathrm{T}$ y $\mathrm{U}$.

Existe una gran diferencia en la representatividad entre las secciones G (comercio al por mayor y al por menor; reparación de vehículos automotores y motocicletas) y C (industrias manufactureras) de algo más del $29 \%$. Dentro de las 268 empresas eficientes de la Sección G, el único grupo que no está es el que corresponde al comercio al por menor en puestos de venta móviles. En la Sección C hay cuatro divisiones, en las cuales no existe ninguna empresa eficiente.
Las secciones I (alojamiento y servicios de comida), S (otras actividades de servicios), D (suministro de electricidad, gas, vapor y aire acondicionado), Q (actividades de atención de la salud humana y de asistencia social), $\mathrm{P}$ (educación) tienen una representatividad individual menor al $1 \%$. Dentro de esta misma Sección, hay 21 grupos de 76 , sin empresas eficientes.

En el sector financiero se destaca la industria aseguradora, pues tiene una participación del $60 \%$ en las entidades eficientes. El subsector cooperativo tiene una representatividad del $0 \%$. El subsector de los bancos, que ocupa el segundo lugar de representatividad después de la industria aseguradora, no alcanzó a tener la cuarta parte de sus entidades dentro del grupo de eficientes. Respecto a la estructura de donde se deriva la eficiencia, en ambos sectores se encuentra una alta concentración en una sola entrada y en una sola salida. No obstante, esta concentración es mayor en el sector real.

Además, es importante analizar el hecho de que, en términos generales, las empresas y las entidades eficientes son muy pocas, apenas llegan a ser el 2,6\% del total de las empresas y entidades estudiadas; de tal manera que es necesario tomar medidas que aseguren una mejora ostensible en su desempeño. Para un país como Colombia, perteneciente a las economías emergentes, es importante que los dos sectores sean eficientes para que se genere desarrollo y las desigualdades sociales se aminoren. 


\section{REFERENCIAS}

Adler, N., Yazhemsky, E. \& Tarverdyan, R. (2010). A Framework to Measure the Relative Socio-Economic Performance of Developing Countries. Socio-Economic Planning Sciences, 44(2), 73-88. https://doi.org/10.1016/j. seps.2009.08.001

Alhassan, A. L. \& Ohene-Asare, K. (2016). Competition and Bank Efficiency in Emerging Markets: Empirical Evidence from Ghana. African Journal of Economic and Management Studies, 7(2), 268-288. https://doi. org/10.1108/AJEMS-01-2014-0007

Almanza-Ramírez, C. (2012). Cost Efficiency in the Banking System in Colombia, 1999-2007: A Non-Parametric Approach. Innovar, 22(44), 67-78.

Andrade, S. (2005). Diccionario de economía. Lima, Perú: Editorial Andrade.

Banker, R. D., Charnes, A. \& Cooper, W. W. (1984). Some Models for the Estimation of Technical and Scale Efficiencies in Data Envelopment Analysis. Management Science, 30(9), 1078-1092. https://doi.org/10.1287/ mnsc.30.9.1078

Cayón, E. \& Sarmiento, J. (2011). Measuring Relative Efficiencies in the Shoe Industry Sector in Colombia: A DEA Approach. Pensamiento \& Gestión, (30), 186-205. Retrieved July 29, 2016, from http://www.scielo.org.co/scielo.php?script=sci_arttext\&pid=S1657-62762011000100010\&lng=en\&tlng=en.

Chan, S. \& Abd Karim, M. Z. (2016). Financial Market Regulation, Country Governance, and Bank Efficiency: Evidence from East Asian Countries. Contemporary Economics, 10(1), 39-54. https://doi.org/10.5709/ce.18979254.197

Charnes, A., Cooper, W. W., Golany, B., Seiford, L. \& J. Stutz (1984). Foundations of Data Envelopment Analysis for Pareto-Koopmans Efficient Empirical Production Functions. In CCS Research Report 504 (pp. 1-28). Austin, TX: Center for Cybernetic Studies, The University of Texas at Austin. 
Apuntes CENES Volumen 36, Número 64

julio - diciembre 2017. Págs. 111-138

Charnes, A., Cooper, W. W., Lewin, A. \& Seiford, L. M. (1994). Data Envelopment Analysis: Theory, Metrodology, and Application. Boston: Kluwe Academic Publishiers. https://doi.org/10.1007/978-94-011-0637-5

Charnes, A., Cooper, W. W. \& Rhodes, E.L. (1978). Measuring the Efficiency of Decision Making Units. European Journal of Operation Research, 2(6), 429-444. https://doi.org/10.1016/0377-2217(78)90138-8

Chiavenato, I. (2004). Introducción a la teoría general de la administración (7 ed.). México: McGraw-Hill Interamericana.

Chortareas, G. E., Garza-García, J. G. \& Girardone, C. (2012). Competition, Efficiency and Interest Rate Margins in Latin American Banking. International Review of Financial Analysis, 24, 93-103. https://doi.org/10.1016/j. irfa.2012.08.006

Cronjé, T. \& de Beer, J. (2010). Combining Efficiency with ROA: Indicator of Future Relative Performance-South African Banking Groups. Corporate Ownership and Control, 7(4 C), 287-296. https://doi.org/10.22495/ cocv $7 \mathrm{i} 4 \mathrm{c} 2 \mathrm{p} 4$

Cotte, A. (2012). Estimating Effectiveness of the Control of Violence and Socioeconomic Development in Colombia: An Application of Dynamic Data Envelopment Analysis and Data Panel Approach. Social Indicators Research, 105(3), 343-366. https://doi.org/10.1007/s11205-010-9772-7

De Oliveira, R. (2002). Teorías de la administración. México: International Thomson.

Estrada, S., Song, H., Kim, Y., Namnb, S. \& Kang, S. (2009). A Method of Stepwise Benchmarking for Inefficient DMUs Based on the Proximity-Based Target Selection. Expert Systems with Applications 36, 11595-11604. https://doi.org/10.1016/j.eswa.2009.03.035

Farrell, M. J. (1957). The Measurement of Productive Efficiency. Journal of the Royal Statistical Society, Series (A), 120(3), 253-281. https://doi. org/10.2307/2343100 
Fontalvo, T., Mendoza, A. \& Visbal, D. (2015). Análisis comparativo de eficiencia financiera: estudio de un caso del sector BASC en Barranquilla. Prospecti$v a, 13(2), 16-24$. Recuperado de https://dx.doi.org/10.15665/rp.v13i2.483

Hahn, F. (2009). A Note on Management Efficiency and International Banking. Some Empirical Panel Evidence. Journal of Applied Economics., 12(1), 69-81. https://doi.org/10.1016/S1514-0326(09)60006-4

Jiang, L., Jiang, Y., Wu, Z., Liao, D. \& Xu, R. (2015). The Measurement of Innovation Efficiency of Chinese High-Tech Industry Using Data Envelopment Analysis. Acta Oeconomica, 65, 101-113. doi:10.1556/032.65.2015.S2.8

Koontz, H. \& Weihrich, H. (2004). Administración. Una perspectiva global (12 ed.). México: McGraw-Hill Interamericana.

Koopmans, T. C. (1951). Analysis of Production as an Efficient Combination of Activities. In T.C. Koopmans (Ed.) Activity Analysis of Production and Allocation (pp. 33-97). New York: John Wiley and Sons.

Li, S. K. \& Zhao, L. (2015). The Competitiveness and Development Strategies of Provinces in China: A Data Envelopment Analysis Approach. Journal of Productivity Analysis, 44(3), 293-307. doi:10.1007/s11123-015-0445-Z

Mankiw, G. (2004). Economía. Madrid: McGraw-Hill Interamericana.

Moreno, J., López, O. \& Díaz, J. (2014). Productividad, eficiencia y sus factores explicativos en el sector de la construcción en Colombia 2005-2010. Cuadernos de Economía, 33(63), 569-588. Recuperado de https://dx.doi. org/10.15446/cuad.econ.v33n63.45347

Nguyen, T. P. T., Nghiem, S. H., Roca, E. \& Sharma, P. (2016). Bank Reforms and Efficiency in Vietnamese Banks: Evidence Based on SFA and DEA. Applied Economics, 48(30), 2822-2835. doi:10.1080/00036846.2015.113 078

Poveda, A. C. (2013). Corruption, Economic Development, and Insecurity in Colombia. In Handbook of Research on Strategic Performance Management and Measurement Using Data Envelopment Analysis (pp. 373-387). https://doi.org/10.4018/978-1-4666-4474-8.ch009 
Apuntes CENES Volumen 36, Número 64

julio - diciembre 2017. Págs. 111-138

Robbins, S. \& Coulter, M. (2005). Administración (8 ed.). México: Pearson Educación.

Rodríguez, G.I. (2003). Medición de la eficiencia relativa en dos subsectores de la economía colombiana desde 1993 a 2002 utilizando Data Envelopment Analysis (DEA). Innovar, 13(22), 121-136. Recuperado de http://www.scielo.org.co/scielo.php?script=sci_arttext\&pi$\mathrm{d}=\mathrm{S} 0121-50512003000200011 \& \operatorname{lng}=$ en\&tlng=es

Rodríguez, G.I. (2016). Indicadores DEA para las actividades de extensión universitaria. Aplicación a la Universidad Nacional de Colombia (2. ${ }^{a}$ ed.). Bogotá: Universidad Nacional de Colombia, Facultad de Ciencias Económicas.

Saberi, M., Mirtalaie, M, Hussain, F., Azadeh, A., Hussain, O. \& Ashjari, B. (2013). A granularcomputing-basedapproachtocreditscoringmodeling. Neurocomputing, 122 100-115. https://doi.org/10.1016/j.neucom.2013.05.020

Samuelson, P. \& Nordhaus, W. (2002). Economía. Madrid: McGraw Hill.

Seffino, M. \& Hoyos, D. (2016). Eficiencia bancaria en Argentina. Comportamiento de los bancos entre 2005 y 2013. Estudios Gerenciales, 32(138), 44-50. Recuperado de https://dx.doi.org/10.1016/j.estger.2015.12.002

Škare, M. \& Rabar, D. (2016). Measuring Economic Growth Using Data Envelopment Analysis. Amfiteatru Economic, 18(42), 386-406.

Superintendencia de Sociedades. (s.f.). Recuperado de http://www.supersociedades.gov.co/Paginas/default.aspx

Superintendencia Financiera de Colombia. (s.f.) Bogotá. Recuperado de: https:// www.superfinanciera.gov.co/jsp/index.jsf

Thanassoulis, E., Boussofiane, A. \& Dyson, R. (1996). A Comparison of Data Envelopment Analysis and Ratio Analysis as Tools for Performance Assessment. Omega, Int. J. Mgmt Sci., 24(3), 229-244. https://doi. org/10.1016/0305-0483(95)00060-7 
Vargas, J.J. (2016). Planteamiento y solución de un modelo DEA estocástico para datos longitudinales con estructura de antedependencia. Bogotá:

Universidad Nacional de Colombia.

Wanga, W., Lu, W. \& Liu, P. (2014). A Fuzzy Multi-Objective Two-Stage DEA Model for Evaluating the Performance of US Bank Holding Companies. Expert Systems with Applications 41, 4290-4297. https://doi.org/10.1016/j. eswa.2014.01.004 